\title{
A new look for Lab Animal
}

Regular readers of Lab Animal will notice that the journal's appearance has changed dramatically this month. Not only has the front cover been redesigned with a new, fresh look, but the inside pages have also been redone and now include additional color and new layouts to improve readability.

So, why the new look? While we have consistently provided the animal research community with high quality news and features, Lab Animal has looked the same since 1998. Having relaunched the Lab Animal website (http://www.labanimal.com) with a new look and increased functionality in the summer of 2004, we wanted to do the same with our print journal to ensure we are presenting our high quality content to our readers in the best way possible.

Aside from the new and improved look, we've made a few small changes to our content. We've introduced 'In This Issue', a new page replacing the 'Editorial'. 'In This Issue' provides a quick overview of key articles in each issue and highlights the page numbers, making it easy for readers to go directly to the articles that they find most relevant or interesting. We have discontinued the 'Supply Lines' column, and in an upcoming issue we will be introducing its replacement, the 'Product Profile' —an 'advertorial' section that offers a forum for manufacturers to introduce new products and services to the laboratory animal science community. Companies wishing to contribute a 'Product Profile' should contact us for more information.

Except for those changes, you'll be happy to know that Lab Animal's content will essentially remain the same. Each issue still contains the mix of monthly columns and peer-reviewed articles you have come to expect from $L a b$ Animal. As always, 'Regulation Watch' keeps you informed on the ever-evolving regulations and guidelines governing the care and use of laboratory animals in the United States and abroad. 'Protocol Review' scenarios get you thinking about ethical dilemmas of the type faced by actual IACUCs. 'What's Your Diagnosis?' case reports continue to present intriguing, atypical animal health situations. In 'Newsfronts', we continue highlighting important and exciting biomedical research findings that resulted from studies using animal models, as well as covering the ongoing struggle between those involved in animal research and the activists that seek to undermine their efforts. Thanks to those of you who contribute papers and serve as peer reviewers, Lab Animal's expert-written feature articles continue to provide timely and practical information that can be applied to improve animal care and husbandry, streamline facility procedures, cut costs, and take the mystery out of facility design and planning projects.

Last spring we conducted a survey among 3,840 of our readers, and a remarkable $31 \%$ responded. The results of this survey gave us some extremely useful insights and information that we will use to determine future content and direction for Lab Animal. Over $96 \%$ of you agreed that the quality of original articles is excellent, and $47 \%$ of our readers read every issue cover to cover. This high level of satisfaction translates into a continually high demand for the journal. That said, it is important to complete your renewals on a regular basis to avoid being dropped from the subscription list.

We continually strive to improve the journal and are always interested in your feedback so please do email us at editors@labanimal.com with any comments or suggestions. We hope you enjoy the new-look Lab Animal! 